\title{
Percutaneous spinal fixation simulation using virtual reality and haptics ${ }^{1}$
}

\author{
Authors \\ Cristian J. Luciano, Ph.D.,1 P. Pat Banerjee, Ph.D.,1-4 \\ Jeffery M. Sorenson, M.D., 9 Kevin T. Foley, M.D., 9 Sameer A. Ansari, M.D., Ph.D., 8 \\ Silvio Rizzl,1 Anand Germanwalla, M.D.6Leonard Kranzler, M.D., 4 \\ Prashant Chittiboina, M.D., M.PH.,7 And Ben Z. RoitberG, M.D., 4.5 \\ Departments of ${ }_{1}$ Mechanical and Industrial Engineering, ${ }_{2}$ Bioengineering, and \\ ${ }_{3}$ Computer Science, College of Engineering, University of Illinois at Chicago; \\ Division of ${ }_{4}$ Neurosurgery, ${ }_{5}$ Radiology, University of Chicago; \\ ${ }_{6}$ Department of Neurosurgery, University of North Carolina, Chapel Hill, NC; \\ ${ }_{7}$ Department of Neurosurgery, Louisiana State University, Shreveport, LA; \\ ${ }_{8}$ Department of Radiology, Neurology, and Neurosurgery, Northwestern University; \\ ${ }_{9}$ Medical Education and Research Institute, Memphis, TN;
}

\section{Corresponding Author}

\author{
Prof. P. Pat Banerjee, Ph.D \\ Mailing Address: \\ Departments of Mechanical and Industrial Engineering, \\ University of Illinois at Chicago \\ $2039 \mathrm{ERF}, \mathrm{M} / \mathrm{C} 251$ \\ 842 W. Taylor, Chicago, IL 60607 \\ Phone 3129965599 \\ Fax: 3124130447 \\ Email:banerjee@uic.edu
}

Acknowledgement: The authors would like to acknowledge the help of Naga Dharmavaram and FeiFei Liu in organizing the data and in running some statistical tests.

\begin{abstract}
Disclosure
Presented at the 2010 AANS annual meeting, this study was sponsored by the AANS Young Neurosurgeons Committee, which does not claim the superiority of the ImmersiveTouch ${ }^{\mathbb{B}}$ system over another system.

The ImmersiveTouch technology has been licensed to ImmersiveTouch, Inc., by the University of Illinois at Chicago.

Coauthor Dr. Banerjee owns stock in ImmersiveTouch, Inc.

The research was supported in part by NIH NIBIB grant 1R21EB007650-01A1

Address reprint requests to: P. Pat Banerjee, Ph.D., University of Illinois at Chicago, Department of Mechanical and Industrial Engineering, 3029 Engineering Research Facility (MC 251), 842 West Taylor Street, Chicago, Illinois 60607. email: banerjee@uic.edu.
\end{abstract}

${ }^{1}$ The research was supported in part by NIH NIBIB grant 1R21EB007650-01A1 


\section{Percutaneous spinal fixation simulation using virtual reality and haptics}

Background. In this study we evaluated the use of a part-task simulator with 3D and haptic feedback as a training tool for percutaneous spinal needle placement.

Objective. The purpose of this study was to evaluate the learning effectiveness in terms of entry point/target point accuracy of percutaneous spinal needle placement on a high-performance augmented reality and haptic technology workstation with the ability to control the duration of computer simulated fluoroscopic exposure, thereby simulating an actual situation.

Methods. Sixty three fellows and residents performed needle placement on the simulator. A virtual needle was percutaneously inserted into a virtual patient's thoracic spine derived from an actual patient computed tomography data set.

Results: Ten out of 126 needle placement attempts by 63 participants ended up in failure for a failure rate of $7.93 \%$. From all 126 needle insertions, the average error (15.69 vs. 13.91), average fluoroscopy exposure (4.6 vs. 3.92), and average individual performance score (32.39 vs. 30.71) improved from the first to the second attempt. Performance accuracy yielded $\mathrm{P}=0.04$ from a two sample $\mathrm{t}$-test in which the rejected null hypothesis assumes no improvement in performance accuracy from the first to second attempt in the test session.

Conclusions: The experiments showed evidence $(\mathrm{P}=0.04)$ of performance accuracy improvement from the first to the second percutaneous needle placement attempt. This result combined with previous learning retention and/or face validity results of using the simulator for open thoracic pedicle screw placement and ventriculostomy catheter placement supports the efficacy of augmented reality and haptics simulation as a learning tool.

\section{KEY WORDS • haptics • neurosurgical simulation • percutaneous spinal fixation • virtual reality}

\section{Background}

At the 2010 annual meeting of the AANS (American Association of Neurological Surgeons), the Young Neurosurgeons Committee continued its annual tradition of organizing a surgical competition using emerging simulators for residents and fellows in the exhibit hall. Percutaneous spinal needle placement was one of the techniques tested, and the results of this testing are reported in this paper. To increase the number of subjects, the same experiment was repeated in January 2011 at the Chicago Review Course on Neurological Surgery, an annual event since 1974 drawing surgeons and residents from all over the world (www.thechicagoreviewcourse.com). The performance of 63 fellows and residents was evaluated for percutaneous spinal needle placement using the head-and-hand-tracked high-resolution and high-performance augmented reality and haptics workstation known as ImmersiveTouch $^{\mathbb{B}}$ (ImmersiveTouch, Inc.). Using a high-definition stereoscopic display and a translucent (half-silvered) mirror, the ImmersiveTouch system ${ }^{1,2}$ superimposes the 3D projection of a virtual instrument over a haptic device stylus, providing force feedback as the trainee performs the virtual surgery on a 3D model obtained from CT and/or MRI of a real patient. An electromagnetic tracking system continuously tracks the position and orientation of the user's head, achieving perfect graphics/haptics collocation as the user moves his/her head, providing the illusion that the surgery is actually performed in a real patient. The system has been evaluated for applications such as ventriculostomy $^{3,4,5,6}$, VP shunt placement ${ }^{7}$ and open thoracic pedicle screw placement ${ }^{8}$. 
Unlike open spinal fixation, in which the surgeon is able to see the bone anatomy, percutaneous spinal fixation requires the surgeon to mainly rely on tactile feedback and fluoroscopy images to guide the proper insertion of the needle through the skin and muscle into the pedicles. Percutaneous spinal fixation needs a different set of skills compared to the equivalent open surgical procedure.

The experiment presented by Luciano et $a .^{8}$ focused on open spinal fixation. It was conducted to study whether the trainees are able to understand the internal spinal structure involved in the procedure, looking at both the 3D model of the exposed spine and multiple views (A/P and lateral) of the fluoroscopy images, as well as CT images, while they insert the needle into the pedicles. During the practice session, the participants were requested to perform multiple insertions with the assistance of fluoroscopy and CT images. After the practice session, the CT and fluoroscopy images were hidden, and therefore, the participants had to remember the skills learnt during the practice session, and perform insertions only based on the appearance of the 3D anatomy and their tactile sensations, but without image guidance at all.

Unlike the previous experiment, the goals of the study reported in this paper (focused on percutaneous spinal fixation), was to determine whether the trainees are able to perform needle insertions with limited image guidance assistance (but without seeing the 3D spine model), trying to focus on their haptic perception to improve their accuracy while minimizing the amount of radiation exposure applied to the patient.

Spinal fixation presents high risks to the patients when performed by inexperienced neurosurgery residents in their early levels of their training, and therefore, there is a prominent need for such simulation. The ImmersiveTouch system offers a number of convenient options for percutaneous needle placement training, allowing the residents to better understand the spinal structure involved in the procedure, and then perform practice in one of the most critical steps in spinal fixation. Other important steps of the procedure, including placement of rod fixation, are less difficult to teach and, therefore, are excluded from the simulation. As shown in Fig 1, the user has the option of monitoring the trajectory of the needle by simulated $\mathrm{A} / \mathrm{P}$, transverse and lateral fluoroscopic views as a means of image guidance. These views can be requested by pressing a foot pedal. The views are available only when the pedal is pressed and the duration of the foot pressing the pedal is recorded to determine the amount of fluoroscopic exposure.

Insert Fig 1 here

The user also has the option to adjust a number of visual and haptic parameters by using a pop up menu as shown in the bottom right of Fig 2. As an example, Fig. 2 shows the same view as Fig. 1 but with the skin of the patient shown in semi transparent mode to clearly see the trajectory of the needle. Depending upon the needs and the skill level of the user, the system can be adjusted to aid the learning process.

Insert Fig 2 here

\section{Objective}

The objective is to experimentally determine learning effectiveness in terms of entry point/target point accuracy of percutaneous spinal needle placement. The user first undergoes training through a practice session. Subsequently the user is asked to repeat the same task that was practiced through a test session. Two attempts are recorded in the test session. The learning effectiveness evaluates comparative performance of the first and second attempts. 


\section{Methods}

\section{Selection Criteria}

Through open solicitations a total of 63 fellows and residents were enrolled as participants. One group was enrolled by the Young Neurosurgeons' Committee at the 2010 AANS annual meeting and the other group was enrolled by Leonard Kranzler at the 2011 Chicago Review Course on Neurological Surgery. The ImmersiveTouch system used for the experiment was provided by the University of Illinois at Chicago, partially supported by ImmersiveTouch, Inc.. A virtual 3D volume of a human spine was created using a CT from a patient at the University of Illinois at Chicago Medical Center. The data were pre-segmented and assembled from a CT DICOM data set after the removal of all identifying personal data. The 3D polygonal isosurfaces corresponding to the skin, and underlying spinal column were extracted.

\section{Experiment}

The participants were given approximately 5 minutes to practice on any pedicle of their choice from among the six pedicles: the left and the right T9, T10 and T11, respectively. During the practice session the participants are taught the importance of needle location accuracy while requesting minimal real-time computer simulated fluoroscopic images in A/P and laterals views, as shown in Fig 1. The fluoroscopic images can be requested by pressing a foot pedal. When the user is satisfied with the final location of the needle, a second foot pedal is pressed to freeze the result. The computer shows the needle location in all two fluoroscopy views (A/P and lateral), as well as a transverse view of the CT image (typically obtained after performing the procedure to verify its outcome). This permitted the users to track all the details of their percutaneous targeting primarily by haptic feedback and periodic image feedback. Following practice the test session consisted of utilizing the skills learnt during practice. The user may then use the cutting tool while moving and rolling the wrist as well as rotating the head to visualize the exact location of the screw and correlate the experience with technique (Fig. 3). For each participant, the final position and orientation of the screw were recorded by the computer.

Insert Fig 3 here

While pushing the needle through the spine, the haptic stylus can be moved along a linear trajectory defined according to its orientation. A reactionary force is applied by the haptic device as the user deviates from that linear trajectory. This is intended to simulate a firm feeling needle placement that is similar to the tactile sensation experienced during surgery. If trajectory is to be changed then the user has to take the needle out of the pedicle first by backtracking on the same linear trajectory before reinserting the needle along a different linear trajectory.

(see Video, Supplemental Video 1, which provides a visual highlight of the percutaneous spinal needle placement simulation, 1 minute, $3.5 \mathrm{MB}$ ) 
Performance data are collected for two successive needle placements. Since both performance error and radiation exposure are equally important to identify whether the percutaneous spinal fixation has been successful or not, two parameters were measured: (i) accuracy in terms of average Euclidean distance from predefined entry and target points, and (ii) duration of fluoroscopic exposure. A cumulative score was computed by giving equal weight to each of these two parameters. The aggregate score is the sum of all individual scores. The lower the score, the better is the performance.

Performance Accuracy Measurement: During the practice and the test sessions the participants were given a recommended landmark on each pedicle to start the needle insertion from and another landmark at which to stop the insertion process. The accuracy score (or performance error) is based on the Euclidean distance in $\mathrm{mm}$ from these targets. If the screw is placed outside of the spinal body, then it carries a penalty of 200 (which is outside of the normal distance range) to the evaluation score to indicate a failure of the procedure.

Fluoroscopic Exposure Measurement: The amount of fluoroscopic exposure is simulated by the duration of foot pedal application in milliseconds. Upon application of the foot pedal the current location of the needle is shown in $\mathrm{A} / \mathrm{P}$ and lateral views.

Failure Rate Measurement: The failure rate is the ratio of the number of failures to the total number of attempts.

\section{Results}

Ten out of 126 needle placement attempts by 63 participants ended up in failure for a failure rate of $7.93 \%$. Thirty three of the 63 participants improved, 28 worsened, and 2 maintained their performance score from the first to the second attempt. From all 126 needle insertions, the average error (15.69 vs. 13.91), average fluoroscopy exposure (4.6 vs. 3.92), and average individual performance score (32.39 vs. 30.71) improved from the first to the second attempt. For performance accuracy, an aggregate Euclidean distance deviation from entry landmark on the pedicle and a similar deviation from the target landmark in the vertebral body yielded $\mathrm{P}=0.04$ from a two sample $\mathrm{t}$-test in which the rejected null hypothesis assumes no improvement in performance accuracy from the practice to the test sessions, and the alternative hypothesis assumes an improvement.

In terms of accuracy, the experiment reported a slightly flatten distribution of the performance error from first to second attempt (Figure 4).

$\underline{\text { Insert Fig } 4 \text { here }}$

Since percutaneous spinal fixation mainly depends on the ability to interpret fluoroscopy images while maintaining the amount of radiation to a minimum to increase patient safety, it is interesting to analyze whether the simulator is able to reduce the amount of fluoroscopy exposure applied by the trainees during the procedure. Comparing the results obtained between first and second attempts, the experiment showed that the participants required a significant lower amount of fluoroscopy shots after a short practice on the simulator (Figure 5).

$\underline{\text { Insert Fig } 5 \text { here }}$ 
Even though the number of needle placement failures during the first and second attempts was the same (5 occurrences), the analysis of the distribution of the final scores, including both performance error and fluoroscopy exposure, showed that the participants were able to improve the outcomes of the virtual surgery during their second attempt (Figure 6).

\section{Insert Fig 6 here}

To assess the impact of duration of requested fluoroscopic image guidance and performance accuracy the null hypothesis is "the change in fluoroscopic exposure use does not associate with the change in accuracy achieved". The results do not show any meaningful correlation between the amount of fluoroscopic image duration request and performance accuracy, hence the null hypothesis cannot be rejected.

The experiment could not correlate scores with level of training as they were not provided by AANS and the Chicago Review Course organizers in order to maintain the anonymity of the participants.

\section{Discussion}

The purpose of this study was to evaluate the accuracy of percutaneous spinal needle placement on a head- and hand-tracked high-resolution and high-performance virtual reality and haptic technology workstation. Specifically we wish to understand the role of virtual reality and haptics simulation in a variety of situations, in this case, percutaneous spinal fixation. The test bed for practicing percutaneous spinal fixation consists of a virtual patient constructed from actual CT scans. A broader goal was to demonstrate the feasibility of using a virtual reality environment as a simulator of spinal neurosurgical procedures by understanding the interplay of various parameters affecting surgical performance, such as accuracy of needle placement and amount of fluoroscopic exposure.

The study demonstrated that the ImmersiveTouch system can be adapted to perform various simulations, and the ubiquitous nature of "entry point/ target point" situations in any invasive procedure. It also demonstrated that computerized fluoroscopic imaging time simulation can be used to help understand the training process.

The accuracy of spinal needle placement achieved by participants using the simulator is comparable to the accuracy reported in a recent retrospective evaluation of such placements ${ }^{9}$. Our results demonstrate significant improvement from one attempt to the next within the virtual reality environment. We plan future studies with greater number of attempts to further explore whether a greater number of attempts will result in greater performance improvement, and at what rate.

Approaches similar in spirit to ours have been reported in literature. For example, Braak, et al. ${ }^{9}$ report a real-time 3D fluoroscopy guidance using cone beam $\mathrm{CT}$ with dedicated needle path planning software as a promising new interventional technique. They assess the accuracy and feasibility of this technique for use in needle interventions. Their approach allows the use of fluoroscopy co-registered with a 3D data set reconstructed from the acquired attenuation information. They project the calculated trajectory on to the real-time fluoroscopy image. However, our approach encapsulates the primary rationale behind such approaches and captures them within the context of a simulator, which 
is much easier to use than such clinical approaches, and at a fraction of the cost. Braak, et al. report fluoroscopy time, accuracy, technical success of the procedures, median procedure time, and complications on 145 interventions on 139 patients. In our simulator we capture the fluoroscopy time, accuracy and procedure time. They report all interventions were within the predefined 5-mm safety margin and achieved $100 \%$ technical success. The median interventional procedure time was 28.5 minutes, and the median fluoroscopy time was 2 minutes 58 seconds. There were minor complications in six patients $(4.3 \%)$ and one major complication $(0.7 \%)$. These statistics are comparable to some of the estimates from our simulator thereby indicating some degree of face validity.

Similarly Tam, et al. ${ }^{10}$ report a retrospective review of early clinical experience of 10 consecutive oncology patients who underwent vertebroplasty of 13 vertebral levels using C-arm cone beam computed tomography (C-arm CBCT) with fluoroscopic overlay for needle guidance during vertebroplasty. Procedural data including vertebral level, approach (transpedicular vs. extrapedicular), access (bilateral vs. unilateral) were recorded. Technical success with the overlay technology was assessed based on accuracy which consisted of four measured parameters: distance from target to needle tip, distance from planned path to needle tip, distance from midline to needle tip, and distance from the anterior $1 / 3$ of the vertebral body to needle tip. Technical success rates were $92 \%$ for both distance from planned path and distance from midline to final needle tip, $100 \%$ when distance from needle tip to the anterior $1 / 3$ border of the vertebral body was measured, and $75 \%$ when distance from target to needle tip was measured. Once again our simulator results bear some face validity to some of these results.

A number of studies using anatomy-specific CT-based finite element methods have been conducted to simulate percutaneous spinal fixation ${ }^{11-14}$, in order to develop a deeper understanding of which vertebrae have the highest risk of fracture or other severe complications. Similarly biomechanical simulations have been conducted to understand the vertebroplasty process better, e.g. cement leakage in percutaneous vertebroplasty ${ }^{15}$. Haptic feedback has been used in some of these biomechanical simulation studies ${ }^{16,17}$ because the physician relies on both sight and feel to properly place the bone needle through various tissue types and densities, and to help monitor the injection of cement into the vertebra. The human-computer interaction for simulating cement injection in such virtual spine workstation is conceptually similar to our approach - however the haptics and problem area studied in this paper are different. In Chui et al. ${ }^{16}$ fluoroscopic images are generated from the CT patient volume data and simulated volumetric flow using a time varying 4D volume rendering algorithm. The user's finger movement is captured by a data glove. Immersion CyberGrasp is used to provide the variable resistance felt during injection by constraining the user's thumb. However such haptics approaches are gradually becoming obsolete because of the advent of superior haptics technology, such as the one used in our approach.

Kobayashi et al. ${ }^{18}$ performed a study to evaluate the accuracy of puncture to the median vertebral body using the unilateral transpedicular approach on percutaneous spinal fixation. They developed a simple puncture simulation method based on the puncture angle determined by preoperative CT. The percutaneous spinal fixation simulator reported in this paper can potentially contribute to validation studies for such approaches.

\section{Conclusions}

Although limited features of our simulator were used in controlled validation experiments to reduce variability in data collection, the capabilities of the simulator go much beyond what is reported here. We have a state-of-the-art percutaneous spinal needle placement simulator that allows experimentation with any patient CT dataset. It uses standard Microsoft Windows platform and common-off-the-shelf controls such as mouse, keyboard, footpedals, Nintendo Wii Remote controls for 3D mouse, and a standard haptic stylus which can hold any physical percutaneous spinal fixation 
needle. In addition the user can adjust material properties of the bone, tissues and skin, can view in transparent or semi transparent mode for ease of learning, can adjust the fluoroscope from continuous, periodic to occasional use settings and can adjust the user background from novice to intermediate to expert settings. The same simulator can be adapted to other percutaneous and open surgical ${ }^{8}$ procedures as well. The greater realism presented in our technology can lead to more effective learning. The experiments showed evidence $(\mathrm{P}=0.04)$ of performance accuracy improvement from the first to the second percutaneous needle placement attempt. The analysis of the average as well as the distribution of accuracy, fluoroscopy exposure and performance score during first and second attempts also showed that the participants improved their outcomes and reduced the risks of complications after a short practice on the simulator. This result combined with the previously published results ${ }^{8}$ of using the ImmersiveTouch simulator for open thoracic pedicle screw placement for learning retention from practice session to the test session supports the efficacy of ImmersiveTouch as a learning tool. Having demonstrated similar efficacy in prior work on ventriculostomy ${ }^{3,5,6}$ and pedicle screw simulations, ImmersiveTouch continues to evolve as a versatile training tool which can be adapted to a variety of situations as needed.

\section{References}

1. Banerjee PP., Luciano C., Florea L. et al. Compact haptic and augmented virtual reality system, U.S. Patent. 2007/0035511 A1, 2010.

2. Luciano C, Banerjee P, Florea L, Dawe G. Design of the ImmersiveTouch ${ }^{\mathrm{TM}}$ : a HighPerformance Haptic Augmented Virtual Reality System. Proc. of Human-Computer Interaction (HCI) International Conf., Las Vegas, July 2005

3. Banerjee PP, Yudkowsky R, Lemole M, Charbel F, Luciano C. Using a High-Fidelity Virtual Reality and Haptics-based Simulation to Determine the "Learning Curve" of Neurosurgery Resident's Surgical Skills. J Simulation in Healthcare. 2007; 2 (2):145.

4. Banerjee PP, Luciano C, Lemole GM Jr, Charbel FT, Oh MY. Accuracy of ventriculostomy catheter placement using a head- and hand-tracked high-resolution virtual reality simulator with haptic feedback. J Neurosurg. 2007; 107:515-521.

5. Lemole GM Jr, Banerjee PP, Luciano C, Neckrysh S, Charbel FT. Virtual Reality in Neurosurgical Education: Part-task Ventriculostomy Simulation with Dynamic Visual and Haptic Feedback. Neurosurgery. 2007; 60(1):142-149.

6. Lemole GM Jr, Banerjee PP, Luciano C, Charbel FT, Oh, MY. Virtual Ventriculostomy with "Shifted Ventricle": Neurosurgery Resident Surgical Skill Assessment Using a High-Fidelity Haptic/Graphics Virtual Reality Simulator. Neurological Research. 2009; 31(4):430-431.

7. Oh M., Banerjee PP, Zhang K, et al. Ventriculoperitoneal Shunt Technique Assessment Using a High-Fidelity Haptic/Graphics Virtual Reality Simulator. Proc. $23^{\text {rd }}$ Int. Computer Assisted Radiology and Surgery (CARS) Congress, Berlin, Germany, 2009

8. C. Luciano, P. Banerjee, B. Bellotte, M. Lemole, M. Oh, F. Charbel and B. Roitberg, "Learning retention of thoracic pedicle screw placement using a high-resolution augmented 
reality simulator with haptic feedback", Neurosurgery , 2011;69(ONS Suppl 1):ons14-ons19, Apr 5 PMID: 21471846

9. Braak SJ, van Strijen MJ, van Leersum M, van Es HW, van Heesewijk JP. Real-Time 3D fluoroscopy guidance during needle interventions: technique, accuracy, and feasibility. AJR Am J Roentgenol. 2010 May;194(5):W445-51. PMID: 20410392

10. Tam AL, Mohamed A, Pfister M, Chinndurai P, Rohm E, Hall AF, Wallace MJ. C-arm cone beam computed tomography needle path overlay for fluoroscopic guided vertebroplasty. Spine 2010 May 1;35(10):1095-9. PMID: 20139803

11. Chae SW, Kang HD, Lee MK, Lee TS, Park JY, The effect of vertebral material description during vertebroplasty. Proc Inst Mech Eng H. 2010;224(1):87-95. PMID: 20225460

12. Wolfram U, Wilke HJ, Zysset PK. Valid micro finite element models of vertebral trabecular bone can be obtained using tissue properties measured with nanoindentation under wet conditions. J Biomech. 2010 43(9):1731-7. PMID: 20206932

13. Wijayathunga VN, Jones AC, Oakland RJ, Furtado NR, Hall RM, Wilcox RK. Development of specimen-specific finite element models of human vertebrae for the analysis of vertebroplasty. Proc Inst Mech Eng H. 2008, 222(2):221-8. PMID: 18441757

14. Kosmopoulos V, Keller TS. Damage-based finite-element vertebroplasty simulations. Eur Spine J. 2004;13(7):617-25. Epub 2004 Jan 17. PMID: 14730441

15. Gisep A, Boger A. Injection biomechanics of in vitro simulated vertebroplasty - correlation of injection force and cement viscosity. Biomed Mater Eng. 2009;19(6):415-20. PMID: 20231794

16. Chui CK, Teo J, Wang Z, Ong J, Zhang J, Si-Hoe KM, Ong SH, Yan CH, Wang SC, Wong HK, Anderson JH, Teoh SH. Integrative haptic and visual interaction for simulation of PMMA injection during vertebroplasty. Stud Health Technol Inform. 2006;119:96-8. PMID: 16404023

17. Lian Z, Chui CK, Teoh SH. A biomechanical model for real-time simulation of PMMA injection with haptics. Comput Biol Med. 2008 Mar;38(3):304-12. PMID: 18078921

18. Kobayashi K, Takizawa K, Koyama M, Yoshimatsu M, Sakaino S, Nakajima Y. Unilateral transpedicular percutaneous vertebroplasty using puncture simulation. Radiat Med. 2006;24(3):187-94. PMID: 16875306

\section{Figure Legend:}

Fig. 1. Percutaneous spinal needle placement with force feedback and the amount of computer simulated fluoroscopic exposure controlled by the time of application of foot pedal

Fig 2. A number of parameters can be controlled by pop-up menus during percutaneous spinal needle placement simulation, for example the skin can be set to semi transparent mode 
Fig. 3. Three-dimensional views of the needle placement for visual analysis of performance

Fig. 4. Distribution of performance error

Fig. 5. Distribution of fluoroscopy exposure

Fig. 6. Distribution of final performance score

\section{Supplemental Content:}

Supplemental Video 1, Video_which provides a visual highlight of the percutaneous spinal needle placement simulation, 1 minute, $3.5 \mathrm{MB}$ ) 


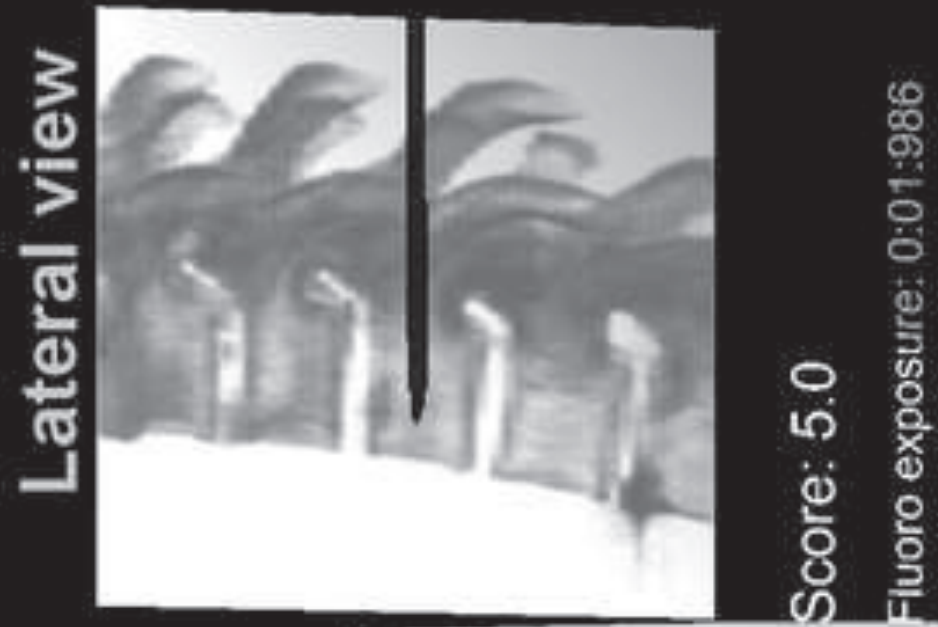

$\frac{10}{60}$

b)

6

.

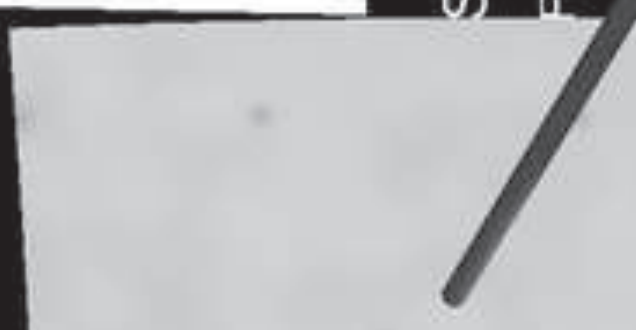

.
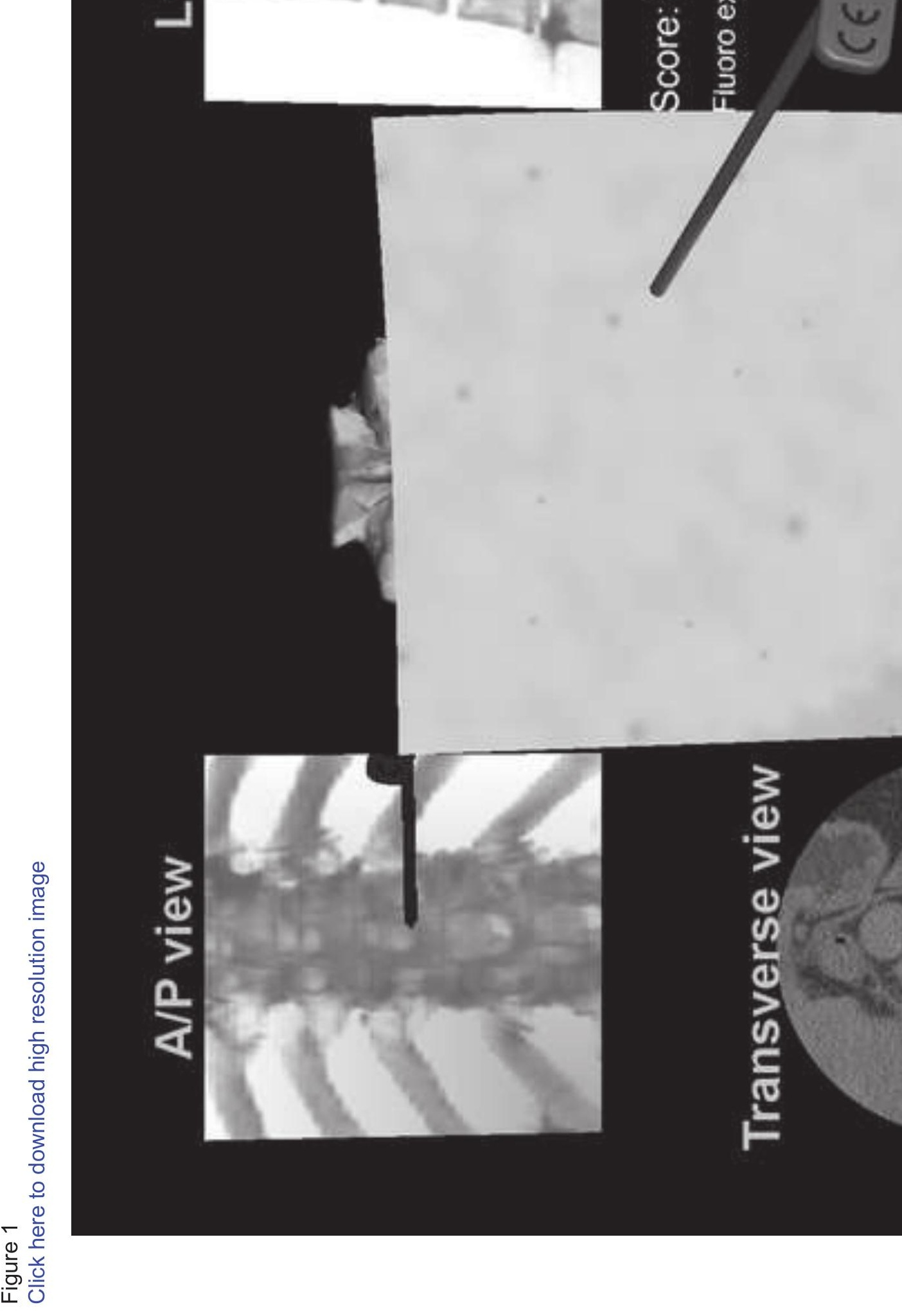

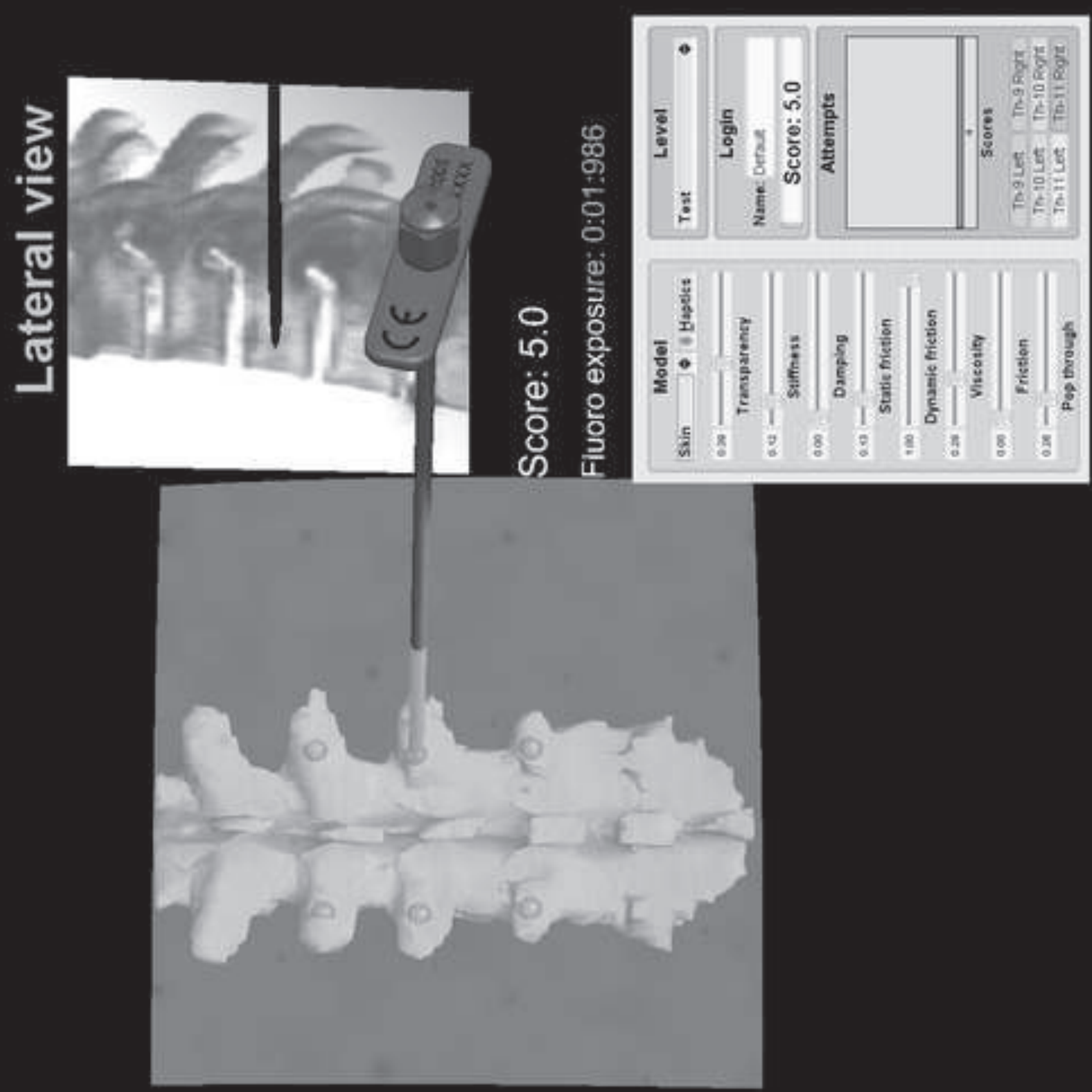

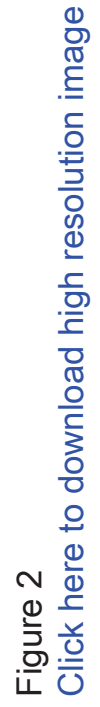
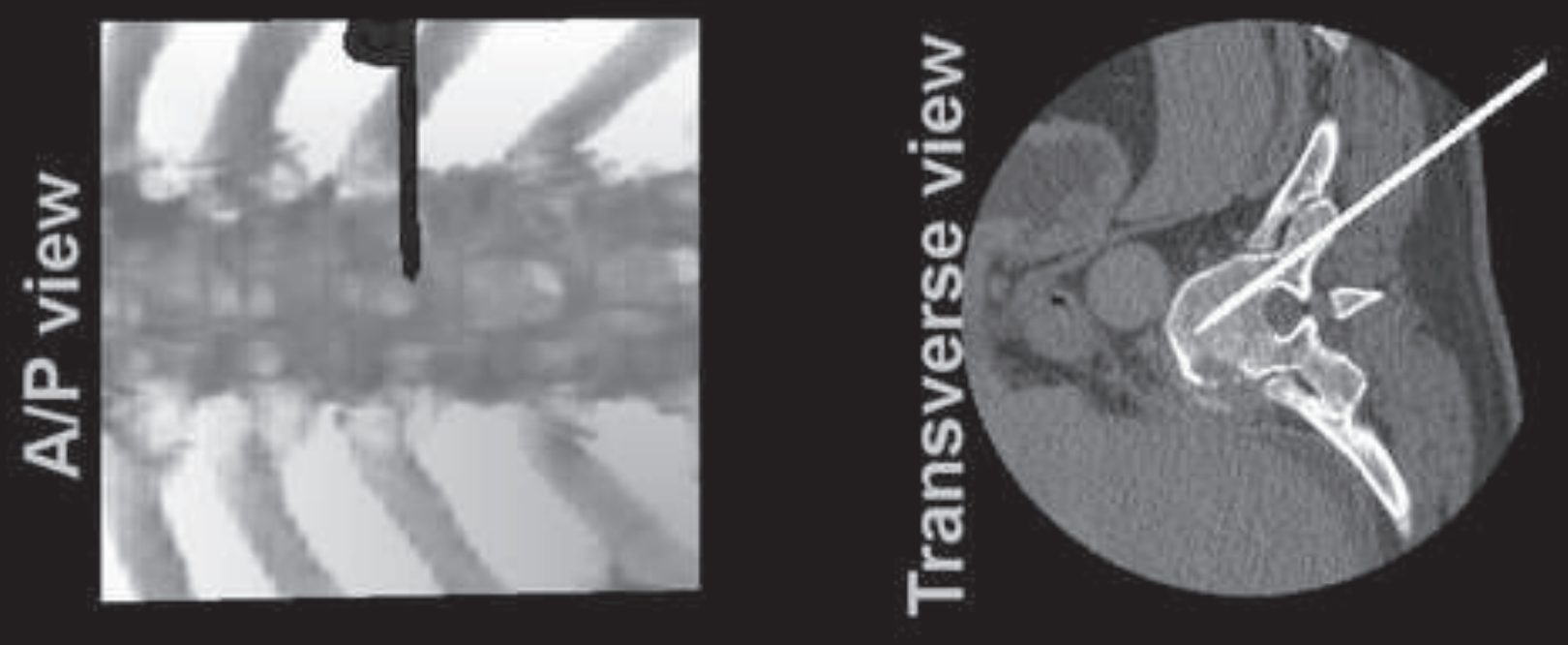

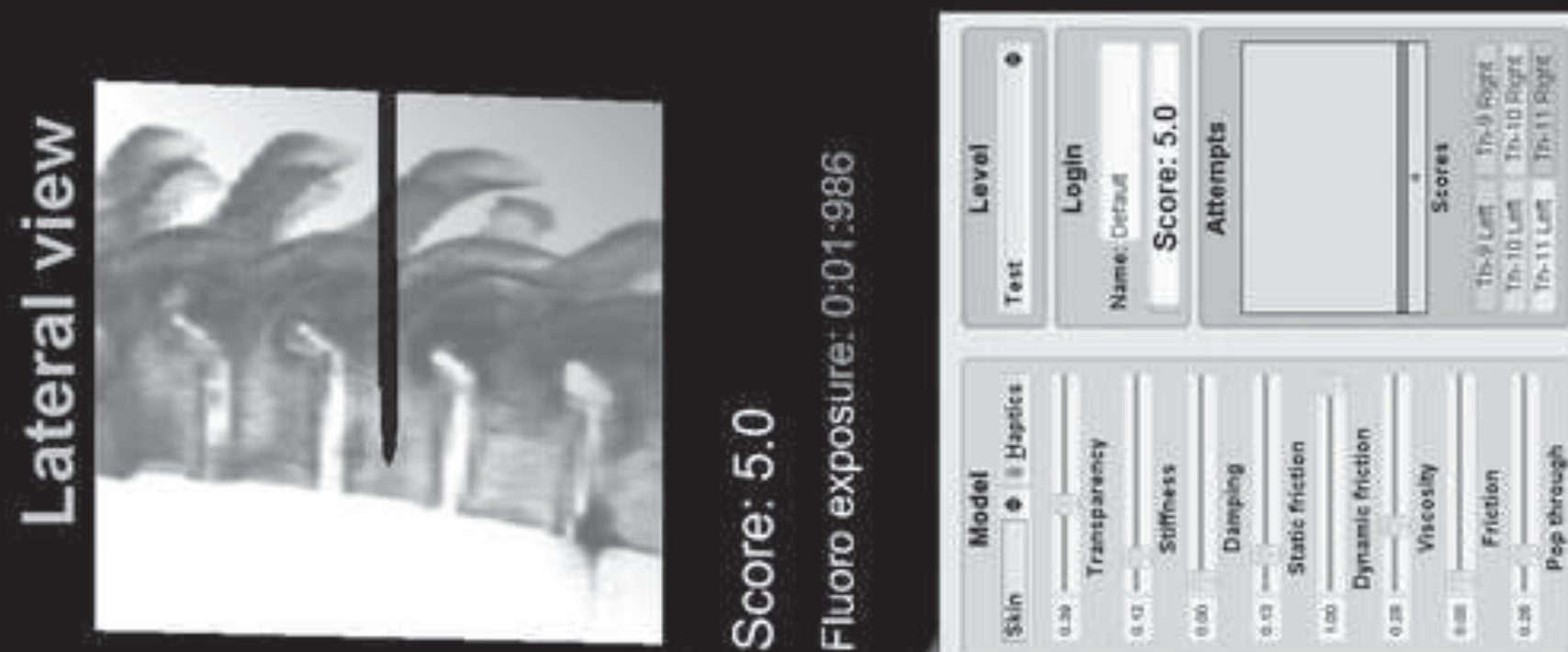

๓
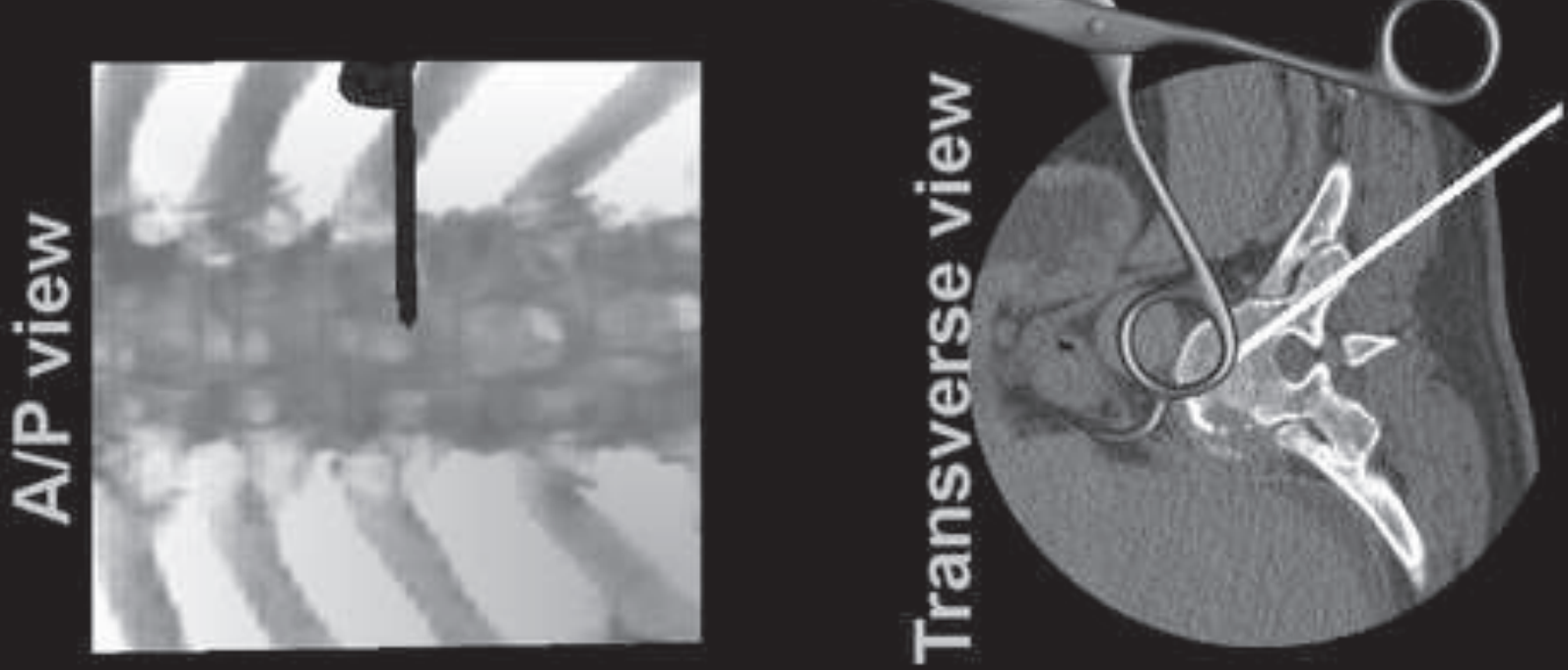


\section{Distribution of performance error}

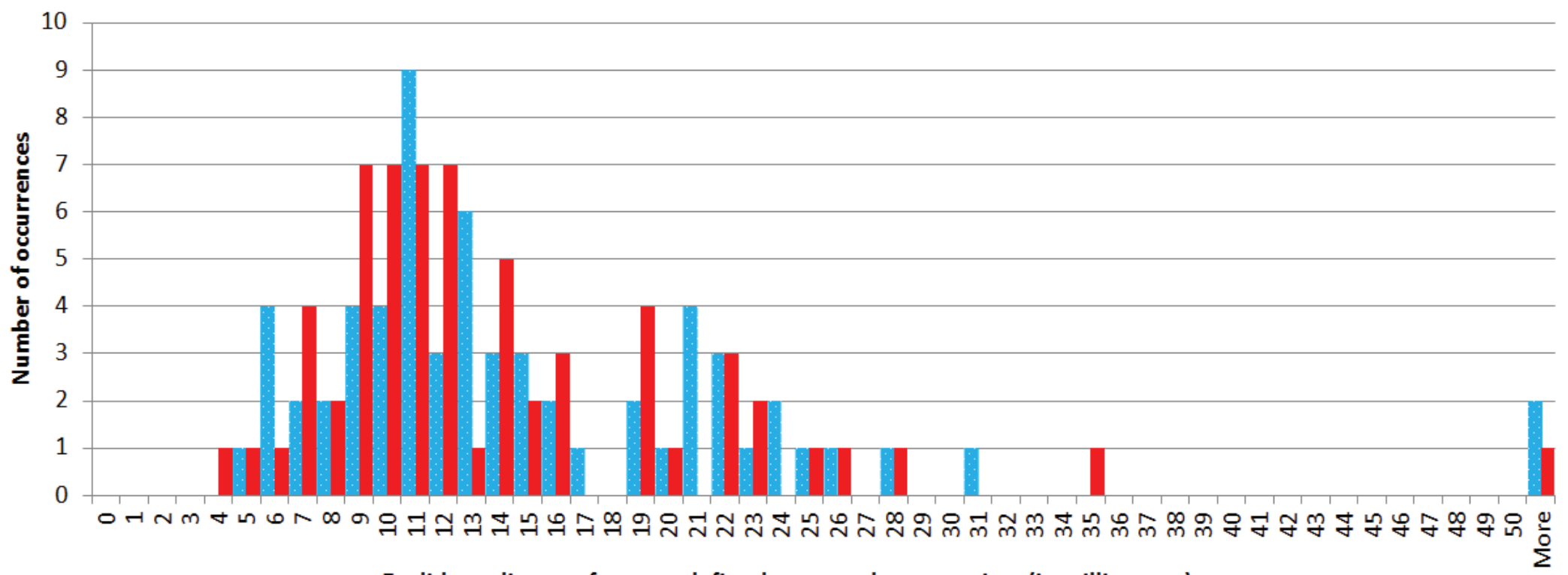

Euclidean distance from predefined entry and target points (in millimeters) 
Distribution of fluoroscopy exposure during first and second attempts

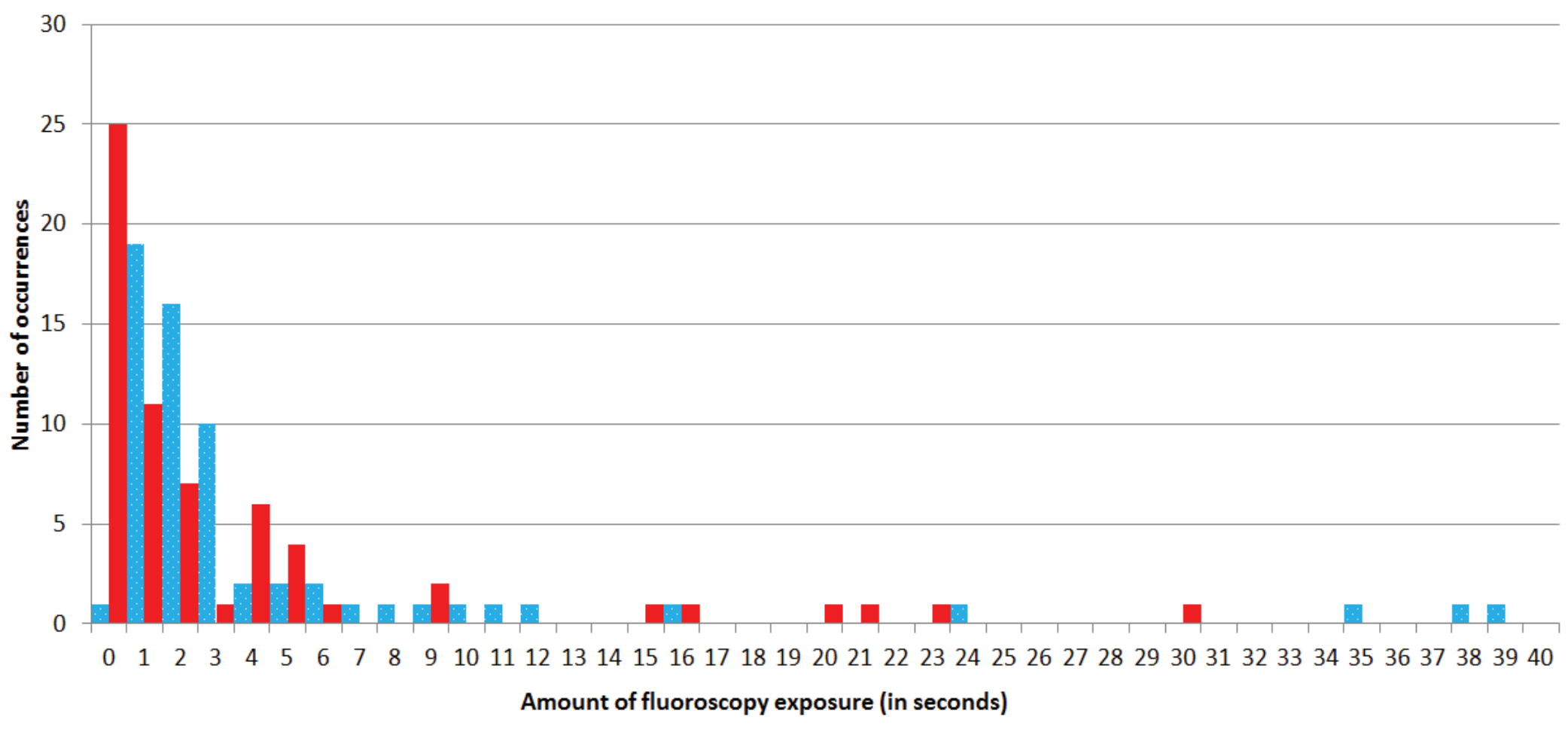


Figure 6

Distribution of final scores

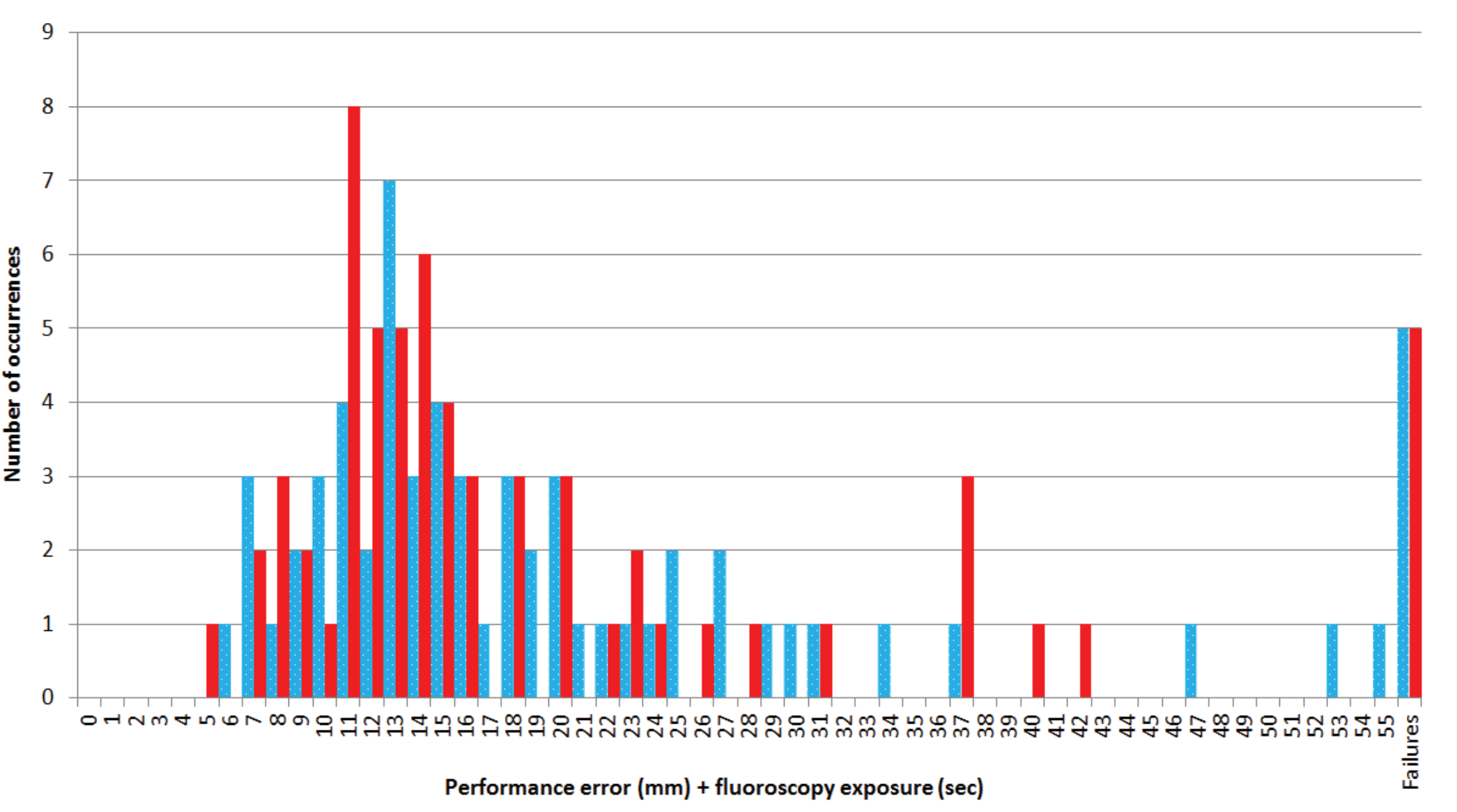

First attempt $\square$ Second attempt 